\title{
Bernardi sul progetto museografico per l'ex Casa del fascio e dell'ospitalità di Predappio
}

Q La playlist dell'intera intervista è disponibile all'indirizzo: https://youtu.be/EQmTK6ZbPQs

Per aprire uno spazio di discussione sull'idea di allestire un museo nazionale sulla storia del fascismo nell'ex Casa del fascio e dell'ospitalità di Predappio, la redazione di "E-Review" ha ritenuto opportuno intervistare Alberto De Bernardi, uno dei membri del Comitato scientifico internazionale che ha affiancato l'Istituto per la storia e le memorie del '900 Parri ER nella fase di progettazione dell'esposizione permanente L'Italia totalitaria. Stato e società in epoca fascista. In questa video-intervista realizzata nel febbraio 2018, gli abbiamo posto una serie di domande volte da un lato a presentare le linee-guida e gli obiettivi del progetto museografico reso pubblico alla fine del 2017, e dall'altro ad affrontare alcune delle obiezioni e delle critiche emerse nel corso del dibattito che negli ultimi anni si è aperto attorno all'iniziativa del Comune di Predappio.

1. Partiamo dal nome della "cosa". Museo? Centro di documentazione? Centro di interpretazione? Quale tra queste è la denominazione più corretta per lo spazio pubblico che si sta progettando?

Ahttps://youtu.be/6EKY1J_G7cs

2. Nella relazione di accompagnamento del progetto museografico sono enunciate le linee-guida per l'allestimento dell'esposizione: proviamo a riepilogarle?

A https://youtu.be/Z5-SEZCzsV8 
3. Nel panorama internazionale le esposizioni museali dedicate alla storia dei totalitarismi sono ormai numerose. Durante la fase di progettazione, avete individuato dei modelli di riferimento?

A https://youtu.be/zPY57ZsGP-E

4. Dopo la presentazione del progetto elaborato dal Comitato scientifico, a che punto è il percorso di allestimento dell'esposizione museografica nell'ex Casa del fascio di Predappio? Quali dovrebbero essere le prossime tappe?

Chttps://youtu.be/HczI4TibfgY

5. Nel dibattito pubblico che si è acceso fin dal momento in cui è stata annunciata l'intenzione di realizzare un museo sul fascismo a Predappio, sono emerse sostanzialmente due principali linee di frattura: una riguarda il luogo, l'altra i contenuti dell'esposizione. In merito al primo punto: perché proprio Predappio? Concordi nel considerare per certi aspetti problematica questa sede? E se sì, come si possono affrontare le criticità?

O https://youtu.be/Bf2DHqf3MxY

6. In merito al secondo punto - e fermo restando che la relazione va a concludere una prima fase dello sviluppo progettuale, volta a delineare la forma d'insieme dell'allestimento e non a stabilire i dettagli di ogni singolo spazio del percorso espositivo - si possono isolare alcuni nodi riguardanti i contenuti della narrazione museale. In particolare, gli aspetti più critici sembrano essere i seguenti: perché uno sguardo generale sull'Italia fascista, e non invece una prospettiva tematica più circoscritta? Soprattutto in certi passaggi (per esempio la sala sulla Mostra della rivoluzione fascista), non c'è il rischio di subordinare il punto di vista dello spettatore a quello dell'autorappresentazione fascista? Rispetto all'intero percorso, non risulta troppo episodica e marginale l'esperienza storica della Rsi? E inoltre, perché è del tutto assente il tema del neofascismo nell'Italia repubblicana?

OAttps://youtu.be/Irdb-acT1vQ

7. Infine, quale rapporto si andrà o si intende instaurare tra questo nuovo luogo di memoria e documentazione storica e la complessiva politica della memoria messa in atto dalla regione Emilia Romagna negli ultimi anni?

A https://youtu.be/EQmTK6ZbPQs 